\title{
Extended Dependency Structures and their Formal Interpretation
}

\author{
Marc Dymetman Max Copperman \\ Rank Xerox Research Centre \\ 6, chemin de Maupertuis, Meylan 38240, France \\ \{dymetman, copperman\} axerox. Er
}

\begin{abstract}
We describe two "semantically-oriented" dependency-structure formalisms, U-forms and S-forms. U-forms have been previously used in machine translation as interlingual representations, but without being provided with a formal interpretation. S-forms, which we introduce in this paper, are a scoped version of U-forms, and we define a compositional semantics mechanism for them. Two types of semantic composition are basic: complement incorporation and modifier incorporation. Binding of variables is done at the time of incorporation, permitting much llexibility in composition order and a simple account of the semantic effects of permuting several incorporations.
\end{abstract}

\section{INTRODUCTION}

U-forms (Unscoped dependency form) are a represen tation formalism which has been used (under a different name) as the basis for the intermediary language in the machine translation system CRIT ILR (Isabelle of al., 1988; Dymetman, 1992; Isabelle, 1995). U-forms account for two central aspects of linguistic structure: predicate-argument relations and headedness (complements vs. modifiers), and so form a middle ground between a "semantic" and a "syntactic" representation. This, combined with their formal simplicity, accounts for much of the popularity of $U$-forms or related formalisms - such as the semantic and deep syntactic representations used in Mel'cuk's Meaning-Text Theory (Mel'čuk, 1981) - in applications such as machine translation and text generation.

Although $U$-forms are strongly "meaning-oriented", their interpretation is never made explicit but is left to the computational linguist's intuition. This has two consequences:

- Operations performed on U-forms and related formalisms cannot be controlled for semantic vat- lidity. So, for instance, it is common practice to define graph rewriting lules on these representations which are believed to produce semantically equivalent expressions. Without the check of formal interpretation, these rules may work in some cases, but produce wrong results in other cases. So for instance, a rule rewriting (the representation of) "John's salary is $\$ 25000$ higher this year than last year" into "John's salary was $\$ 2,5000$ lower last year than this year" would seem in tuitively valid until one considered the case of "John's salaty is $50 \%$ higher this year than last yeat". where it does not work any more.

- U-forms are not directly adapted to applications putting emphasis on denotational semantics and formal reasoning, like for instance some natural language generation systems in well-formalized domains (Fuang and Fiedler, 1995; Ranta, 1995; I evine, 1990), see also (Alshawi, 1992).

A basic obstacle to providing a formal interpretation for U-forms is the fact that these representations leave the relative scopes of dependents implicit. The $S$-form representation (Scoped dependency form), which we introduce here, is an extension of U-form notation which makes scope explicit, by allowing dependents to be ordered relative to one another. Dependents (complements or modifiers) can move freely relative to one another in the S-form structure, under certain binding-site constraints.

We then go on to provide a compositional interpretation mechanism for $S$-forms. Free variables (generalizations of the $\arg _{1}$, $\arg _{2}, \arg _{3}$ annotations of standard dependency formalisms) are used to connect an argument to its binding-site inside a predicate. Binding of variables is done at the time of incorporation, permitting much flexibility in composition order and a simple account of the semantic effects of permuting several incorporations. This liberal use of free variables is contrasted to the approach of Montague grammar, where the requirement that semantic expressions entering into a composition are closed (do not contain 
free variables) leads to a certain rigidity in the order of composition.

Two kinds of semantic composition are basic: com plement incorporation. where the complement fills a semantic role inside the head, and modifier incorporation. where the head fills a semantic role inside the modifier. The mechanism of actually deriving the semantic translation of the composition from the scmantic translations of its two components is handled through a list of type-sensitive composition rules. which determine the action to be taken on the basis of the component types. The flexibility of the approach is illustrated on an example involving proper names. quantified noun phrases, adverbials and relat tive clauses.

\section{U-FORMS}

Formally. U-forms are unordered labelled n-ary trees such as the one shown in Fig. 1, comresponding to the sentence: (S1) "John does not like every woman hated by Peter".

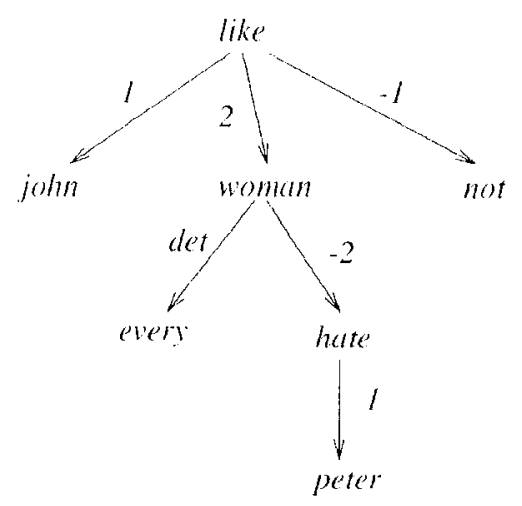

Figure 1: A U-form.

The edge labels are members of the set $\{$ det, $1,2,3$, $\ldots,-1,-2,-3, \ldots\}$, and correspond either to determiners (label 'det') or to argument positions relative to a predicate node (other labels).

The U-form of Fig. 1 expresses three predicalteargument relations among the nodes:
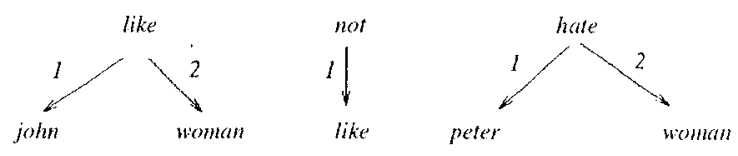

Figure 2: Predicate-argument relations in a U-form.

In order to extract the predicate-argument relations encoded into the U-form, one needs to apply the following "rule". Let's notate $(A, L, B)$ an edge of the tree, where $A$ is the upper vertex. B the lower vertex, and $L$ the edge label. With each node $A$ in the tree, one associates its set of predicution edges, that is the set $P \Lambda_{A}$ of edges of the form $\left(A_{,}+i . X\right)$ or $(X$.$\mathrm{i}, \mathrm{A}$ ). One then considers the predication tree $\mathrm{T}_{1}$ made by forming the collection of edges $(\Lambda, I, X)$ where $I$. is positive and either (A.L.X) or (X.inverse (L).A) is a predication edge of $A$. Each predication tree denotes a predicite-argument relation among U-form nodes. So for instance. the tree $\mathrm{l}_{\mathrm{f} \text { rate }}$ is formed by forming the edges (hate, 1,peter) and (hate.2.woman). and this corresponds to the predicate-argument relation hate(peter.woman).

WELL-FORMEDNESS CONIDITIONS ON UFORMS In order to be well-formed. a L-form LI: has to respect the following condition. For any node A of UF, the predication tree $T_{A}$ nust be such that:

1. No holes condition] If (A.i.B) is an edge of ' $\mathrm{T} .1$. then for any number $\mathrm{j}$ between 1 and $i$. T. I must contain a node of form $(\Lambda . j . C)$.

2. [No repetition condition $\mid$ No two edges of ' $\mathrm{I}_{1}$ can have the same label $i$.

MORE ON U-FORMS Negative labels ate a device which permits to reconcile the notation of predicateargument structure with the notation of syntactic dependency. So, in the U-form considered above, while "semantically" the "woman" node is an argument of the "hate" node. "syntactically" the 'hate" node is a dependent of the "woman' node. Cases such as this one. where there is a conflict between predicate-alrgument directionality and dependency directionality are notated in the U-form through negative labels, and correspond to modifiers. Cases where the directionality is parallel correspond to complements.

When used as interlingual representations in machine translation systems, U-forms have several advantages. The first is that they neutralize certain details of syntactic structure that do not carry easily between languages. For instance. French and English express negation in syntactically different ways: "Rachel does not like Claude" vs. "Rachel n' aime pas Claude": this difference is neutralized in the U-form representation, for both negations are expressed through a single negation predicate in the $U$-form.

A second advantage is that they represent a good compromise between paraphrasing potential and se mantic precision. So. for instance, in the CRITTER system. the three sentences:

John does not like every woman that Peter hates John does not like every woman hated by Peter

Every woman whom Peter hates is not liked by John

would be assigned the U-form of Irig. 1. On the other hand, the sentence: 
Peter hates every woman that John does not like

would be assigned the U-form of lig. 3, which is different from the previous U-form, although the predicate-argument relations are exactly the same in both cases.

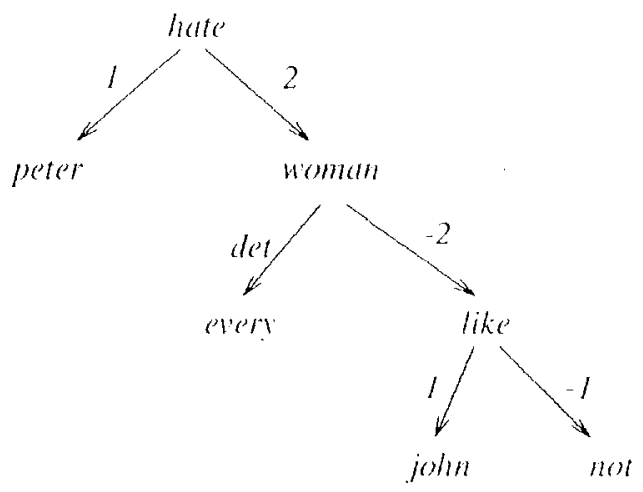

İgure 3: $A$ different U-form.

One can take alvantage of such paraphrasing poten tial in certain cases of syntactic divereence between languages. For instance. French does not have a syntactic equivalent to the dative-movenent + passive configuation of:

\section{Rachel was given a book by Claude}

so that a direct syntactic translation is not possible. However, at the level of $\mathrm{U}$-lorm, this sentence is equivalent to the Irench sentence:

\section{Claude a donné un livre à Rachel}

and this equivalence can be exploited to provide a translation of the first sentence.

One serious problem with $U$ forms, however, is that they do not have unambiguous readings in calses where the relative scopes of constittents can result in different semantic interpretations. So, in the case of sentence (S1), the two readings: "it is not the case that John likes every woman hated by Peter", and "John dislikes every woman that Peter hates" are not distinguished by the U. form of lig. 1 .

\section{S-FORMS}

INTRODUCING SCOPE Let's consider the trec represented in Tig. 4

The only difference between this tree and the $\mathrm{I}$ form of lig. 1 is that the nodes of our new tree are considered ordered whereals they were considered unordered in the U-form. The convention is now that dependent sister nodes are interpreted as having different scopes. with narower scope cotresponding to a position more to the right.

The tree of lig. 4 can be glossed in the following way:

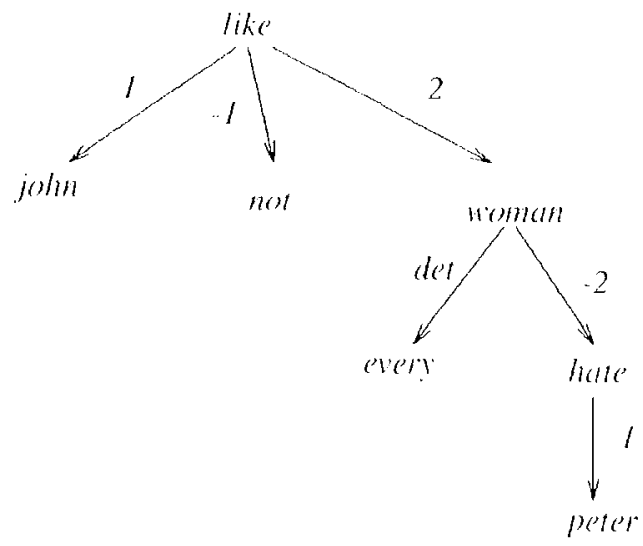

Figure 4: Introducing scope by ordering the nodes

John, it is not the case that he likes every woman that $\mathrm{Pe}$ ter hates

If we consider the six permutations of the nodes under like. we can produce six different scopings. Because John refers to an individual. not a quantified NP. these six permutations really correspond to only the two interpretations given above. The tree of Fig. 4 corresponds to the first of these interpretations. which is the prefered interpreation for sentence (Si).

Our discussion of scope being represented by node order has been informal so far. In order to make it formal, we need to encode our representation into a binary-tree format on which a compositional semanties can be defined. To do thatt. in a first step we replate the argument numbers of lig. 4 by explicit argument names; in a second step we encode the resulting or dered n-ary tree into a binalry format which makes ex plicit the order in which dependents are incorporated into their head.

S-RORMS Consider the n-ary tree of lig. f. For any node $A$ in this tree, take the set of predication edges associated with $A$, that is the set of edges $\left(A+i, B_{i}\right)$ and $\left(B_{i},-i, \Lambda\right)$. By renaming each such node $\Lambda$ into $\mathrm{A}\left(\mathrm{X}_{1}, \ldots, \mathrm{X}_{n}\right)$, where $\mathrm{X}_{1}, \ldots, \mathrm{X}_{n}$ are tresh identifiers. and by renaming each such label $+i$ (resp. $-i$ ) into $+X$, (resp). $-X_{i}$ ), one obtains a new tree where argument numbers have been replaced by argument names. For instance the previous representation now becomes the tree of lïg. 5.

This representation is called a scoped dependency form, or $S$-form.

BINARY TREE ENCODING OF S-FORMS: BFORMS In order to encode the ordered n-ary tree into a binary tree, we need to apply recursively the transformation illustrated in Fig. 6, which consists in forming a "head-line", projecting in at north-west direction from the head $I I$, and in "attaching" to this line "dependent-lines" $\mathrm{D}_{1}, \mathrm{D}_{2}, \ldots, \mathrm{D}_{n}$, with $\mathrm{D}_{1}$ the right- 


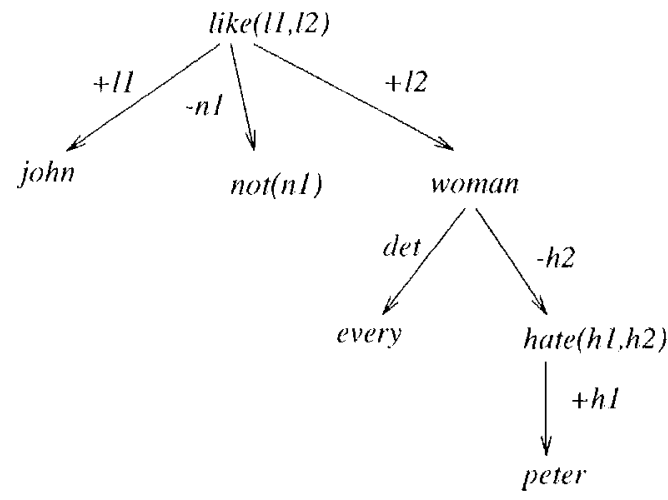

Figure 5: An S-form.

most dependent (narrowest scope) and $\mathrm{D}_{n}$ the leftmost dependent (widest scope) in the original tree.
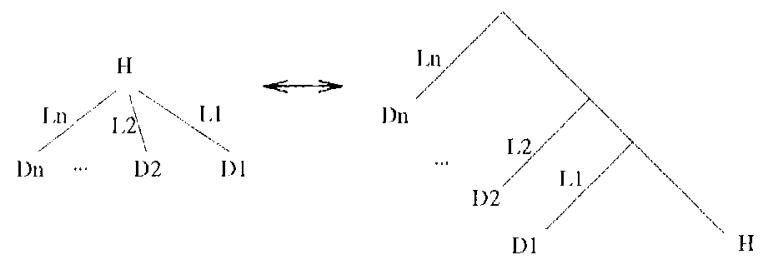

Figure 6: The transformation between S-forms and Bforms.

Applying this encoding to our example, we obtain the binary tree of Fig. 7, which is called a B-form.

The B-form makes explicit the order of incorporation of dependents into the head-line. By permuting several dependent-lines along their head-line, this incorporation order is changed and gives rise to different scopings.

S-forms and B-forms are completely equivalent representations. Clearly, the encoding, called the $S$ form/ $B$-form encoding, which has just been defined is reversible. The $S$-form is more compact and makes the dependency relations more conspicuous, whereas the B-form makes the compositionality more explicit.

WELL-FORMEDNESS CONDITIONS ON BFORMS AND S-FORMS Starting from the U-form and enriching it, we have informally introduced the notions of S-form and B-form. We now define them formally.

We start by giving a recursive definition of IBFs (incomplete B-forms), that is, B-forms which may contain unresolved free variables. We use the notation ((D,Label), H) the labelled binary tree obtained by taking $\mathrm{H}$ as the right subtree, D as the left subtree, and by labelling the left edge with Label. We also use the notation fv(IBF) for the set of the free variables in IBF

\section{DEFINITION OF INCOMPLETE B-FORMS}

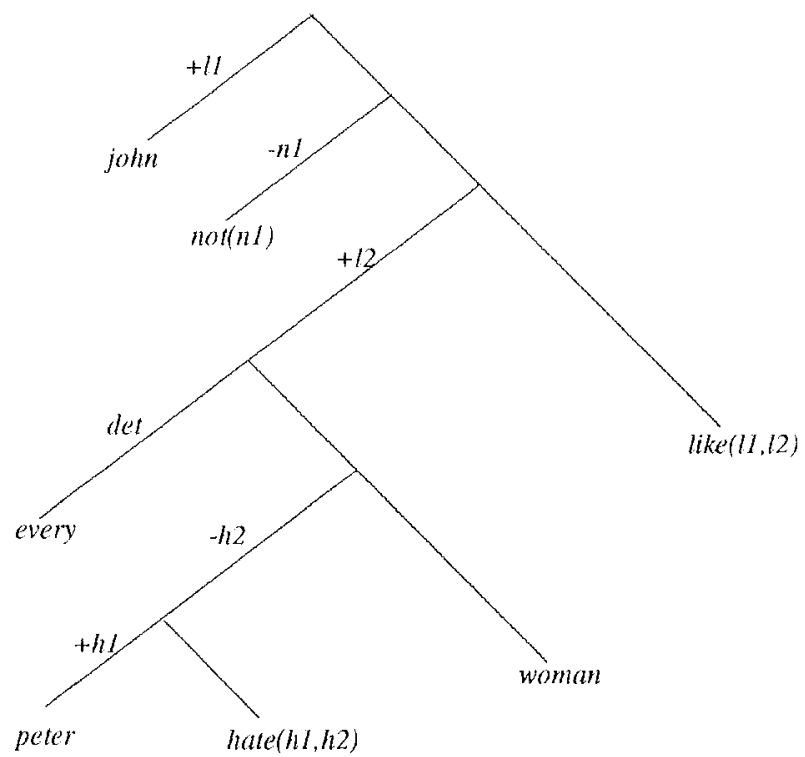

Figure 7: A B-form.

1. A node $\mathrm{N}$ of the form $\operatorname{Pred}(\mathrm{x} 1, \ldots, \mathrm{xn})$ is an $\mathrm{IBF}$ with the set of free variables $f v(N)=\{x 1, \ldots, x n\}$;

2. If $\mathrm{D}$ and $H$ are IBFs, $f v(D)$ and $f v(H)$ are disjoint, and $x \in f v(H)$ then $H^{\prime}=((D,+x), H)$ is an IBF with $f v\left(H^{\prime}\right)=f v(D) \cup f v(H) \backslash\{x\} ;$

3. If $D$ and $H$ are IBFs, $f v(D)$ and $f v(H)$ are disjoint, and $x \in f v(D)$ then $H^{\prime}=((D,-x), H)$ is an $I B F^{\prime}$ with $f v\left(H^{\prime}\right)=f v(D) \cup f v(H) \backslash\{x\}$;

4. If $D$ and $H$ are IBFs, and $f v(D)$ and $f v(H)$ are disjoint, then $H^{\prime}=((D, d e t), H)$ is an IBF with $f v\left(H^{\prime}\right)$ $=\mathrm{fv}(\mathrm{D}) \cup \mathrm{fv}(\mathrm{H})$.

DEFINITION OF B-FORMS A B-form is an IBF with an empty set of free variables.

The notion of S-form can now be defined through the use of the $S$-form/B-form encoding.

DEFINITION OF S-FORMS A S-form is an ordered labelled $n$-ary tree which can be obtained from a B-form through the inverse application of the S-form/Bform encoding.

It can be easily verified that the representation of Fig. 7 is indeed a B-form, and, consequently, the representation of Fig. 5 is a valid S-form. More generally, it can be easily verified that enriching a U-form by ordering its nodes, and then replacing argument variables by argument names always results in a valid $S$ form. ${ }^{1}$

\footnotetext{
${ }^{1}$ The converse is not true: not all S-forms can be obtained in this way from a U-form. For instance, there exists a $S$-form conresponding to the preferred reading for "Fido visited most trashcans on every street", which has "every street"
} 


\section{THE INTERPRETATION PROCESS}

We now describe the interpretation process on B-forms. Interpretation proceds by propagating semantic translations and their types bottom-up.

The first step consists in typing the leaves of the tree, while keeping track of the types of free variables, as in Fig. 8.

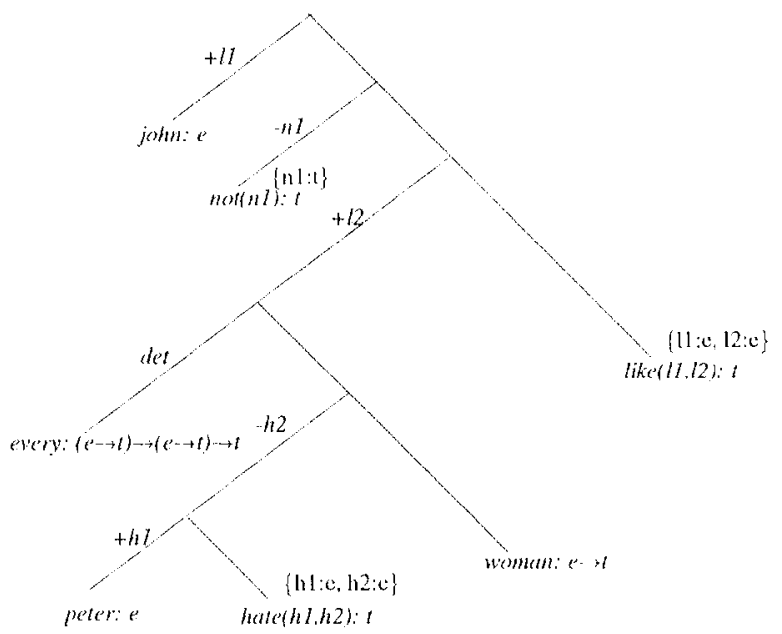

Iigure 8: Typing the leaves. The free variables and their types are indicated in brackets.

The types given to the leaves of the tree are the usual functional types formed starting with e (entities) and t (truth values). In the case where the leaf entity contains free variable arguments, the types of these free variables are indicated, and the type of the leaf takes into account the fact that these free variables have already been included in the functional form of the leaf. Thus hate(h1,h2), which can be glossed as: "h 1 hates $h 2$ ", is given type $t$, while $h 1$ and $h 2$ are constrained to be free variables of type $e$.

VARIABLE-BINDING RULES According to the well-formedness conditions for B-forms, a complement incorporation $((\mathrm{D},+\mathrm{x}), \mathrm{H})$ is only possible when $H$ contains $x$ among its free variables; the "syntactic dependent" $D$ is seen as semantically "filling" the place that $x$ occupies in the "syntactic head" $H$. In the same way, a modifier incorporation $((\mathrm{D},-\mathrm{x}), \mathrm{H})$ is only possible when $D$ contains $x$ among its free variables;

outscoping "most trashcans", and which is not obtained from a U-lorm in this simple way. However, there exists a mapping from S-forms to U-lorms, the scope-forgetting mapping, which permits to define equivalence classes among $\mathrm{S}$ forms "sharing" the same U-form. This relation between $\$$ forms and $U$-forms can be used to give a (non-deterministic) formal interpretation to U-forms, by considering the interpretations of the various S-forms associated with it (see the technical report companion to this paper.) in this case the "syntactic" head $I I$ is seen as semantically "filling" the place that $x$ occupies in the "syntactic dependent" $D$. (This difference corresponds to the opposition which is sometimes made between syntactic and semantic heads and dependents: complements are dependents both syntactically and semantically, while modifiers are syntactically dependents but scmantically heads.)

In order to make formal sense of the informal notion "filling the place of $x$ in $A_{x}:$ " (where the notation $\Lambda_{x}$ means that $\mathrm{A}$ contains the free variable $\mathrm{x}$ ), we introduce the variable-binding rules of Fig. 9 .
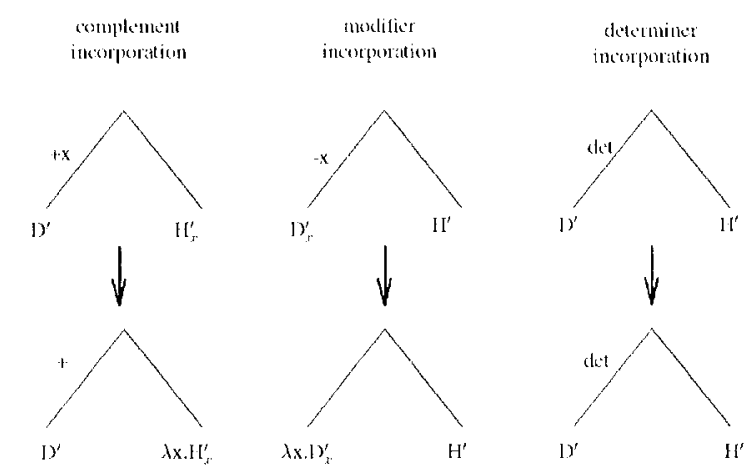

Figure 9: Variable-binding rules. D' and H' correspond to the semantic translation of the subtrees rooted in $\mathrm{D}$ and $\mathrm{H}$ respectively.

These rules tell us how to "get rid" of the free variable being bound during complement or modifier incorporation, namely by forming the abstraction $\lambda x . \mathrm{A}_{x}$. before actually performing the semantic composition between the dependent and the head. For completeness, determiner incorporation, which does not involve variable binding, is given along with complement and modifier incorporation.

Two things should be noted about this way of "delaying" variable-binding until the relevant dependent is incolporated:

- Suppose that we had bound the variables appearing in the head predicate locally, that is to say, that, in the style of Montague grammar (Camut, 1991), we had written $\lambda 1211$.like $(11,12)$ instead of like $(11,12)$, and so forth, in Fig. 7. Then each incorporation of a dependent into the "head-line" would have changed the type of the head; thus 'not' would have had to combine either with a head of type $\mathrm{e} \rightarrow \mathrm{e} \rightarrow \mathrm{t}$, or $\mathrm{e} \rightarrow \mathrm{t}$, or $\mathrm{t}$, depending on its scope relative to the other dependents; with the scheme adopted here, the type of the head remains invariant along the head-line;

- Under the same hypothesis, the incorporation of the second argument first and of the first argument second would have been much simpler than 
the reverse incorporation order. and some mechanism would have had to be found to distinguish the two orders. Then permuting the relative order of two dependents along the head-line - corresponding to different scope possibilities - would have had complex computational consequences. In the scheme adopted here. these cases are handled in a uniform way.

The way free variables are used in our scheme is somewhat reminiscent of the use of syntactic variables he, in Montague grammar. Montague grammar has the general requirement that only closed lambda-terms (lambda terms containing only bound variables) are composed together. This requirement. however. is difficult to reconcile with the flexibility needed for handling quantifier scope ambiguities. Syntactic variables are a device which permit to "quantify into" clauses at an arbitrary time, bypassing the normal functional composition of lambda-terms. which requires a strict management of incorporation order. In our scheme. by contrast. this secondary mechanism of Montague grammat is graduated to a central position. Composition is always done between two lambda-terms one of which at least contains a free variable which gets bound at the time of incorporation.

TYPE SENSITIVE COMPOSITION RULES If we apply the variable-binding rules to the subtree $\mathrm{PH}=(($ peter. $-\mathrm{h} l) \cdot$ hate $(\mathrm{h} l, \mathrm{~h} 2))$ of Fig. 8 . we find that we must compose the semantic translations peter and $\lambda$ h1 hate (h1.h2) in "complement" $(+)$ mode. The first function is of type e, while the second function is of type $\mathrm{e} \rightarrow \mathrm{t}$ (for hate $(\mathrm{h} 1, \mathrm{~h} 2$ ) is of type $t$. and $\mathrm{hl}$ of type c).

How do we compose two such functions? A first solution, in the spirit of Lambek calculus (Morrill. 1994) or of linear logic (Dalrymple et al. 1995), would be to define a general computational mechanism which would be able. through a systematic discipline of typechanging operations. to "adapt" automatically to the types of the functions undergoing composition.

Such mechanisms are powerful, but they tend to be algorithmically complex, to be non-local, and also to give rise to spurious ambiguities (superficial variations in the proof process which do not correspond to different semantic readings).

Here, we will prefer to use a less general mechanism. but one which has two advantages. First, it is local, simple, and efficient. Second, it is flexible and can be extended to handle the semantics of sentences extracted from a real corpus of texts, which it might be perilous to constrain too strongly from the stat.

The mechanism is the following. We cstablish a list of acceptable "type-sensitive composition rules", which tell us how to compose two functions according to their types. Such a (provisory) list is given below:"

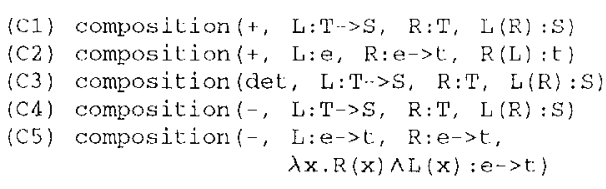

The entries in this list have the following format. The first argument indicates the type of composition ('+' for complement incorporation. -' for modifier incorporation. "det' for determiner incorporation): the second argument is of the form Left:I eft lype. where Left is the felt transtation entering the composition. and Left'lype is its type: similarly, the second argement Right:Right Type corresponds to the right subtree entering the composition: finally the third argument gives the result Result:Result lype of the composition. where the notation $A(B)$ has been used to indicate standard functional application of function $A$ on areument B. Uppercase letters indicate unifiable variables.

It may be remarked that if, in these rules. we neglect the functions themselves (I.eft. Right. Result) and concentrate on their types (IefiType, RightType. ResultType). then the rules can be seen as imposing constraints on what can count as validly typed trees: these constraints caln flow from mother to daugthers as well as in the opposite direction. Thus. through these rules. knowing that the head-line functions projecting from a verbal head must be of type t imposes some constraints on what are the possible types for the dependents: this can be useful in particular for constraining the types of semantically ambiguous lexical elements.

If we now go back to our example. we have $10 \mathrm{com}-$ pose in complement mode $(+)$ the function peter, of type e. with the function $\lambda h 1$ hate(h1.h2). of type $\mathrm{e}-\mathrm{t}$. Consulting the list of composition rules. we see that the only applicable rule is (C2). and that the result is $\lambda$ h1 hate $($ h $1 . h 2)($ peter $)=$ hate $($ peter.h2), of type 1 .

Now that we have the semantic translation hate(peter.h2) for the subtree PH, we can compute the translation for the subtree ((PH,-h2),woman). By the variable-binding rule for modifiers. we need first to form the abstraction $\lambda$ h2.hate(peter:h2). of type $\mathrm{e} \rightarrow \mathrm{t}$. and compose it in "-" mode with woman. of type e-t. Consulting the list of composition rules. we find that the only applicable rule is ( $\mathrm{C} 5$ ). and that the result of this application is $\lambda$ h2.woman(h2)/hate(peter.h2). ${ }^{3}$

It is a matter for further research to propose principles for producing such rules. Some of them can be seen as special cases of general type-raising principles. others (such as C5) are necessary if one accepts that the type of intersective adjectives and restrictive relative clauses has to be $e-\rightarrow$.

The rule (C5) differs from the previous rules in the list in that it introduces the logical connective $\wedge$ which does not originate in lunctional material already present in either of the arguments. A possible justification for the rulc. however, 


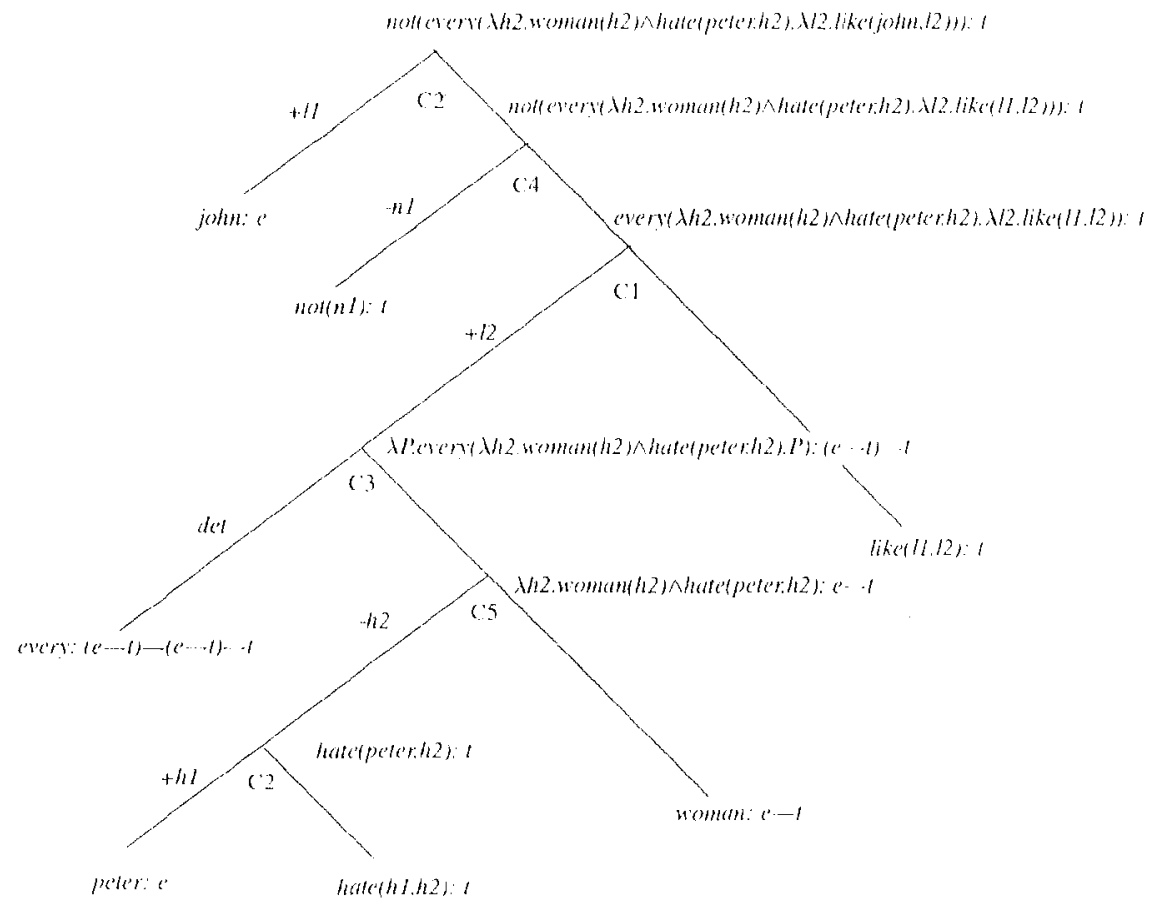

liggure 10: B-form interpretation. Lor every'. we make use of the gencralized quantitier notation quant(restrictionsesope).

The process of semantic translation proceeds in this way bottom-up on the B-form. The end result is shown in fig. 10 .

\section{Acknowledgments}

Thanks to Alain I ecomte and Fédérigue Segond for comments and discussions.

\section{References}

Hiyan Alshawi. editor. 1992. The Core Language Lingine. 'The MI'T' Press.

J. Barwise and R. Cooper. 1981. Generalized quantifiers and natural language. Linguistics and Philosophy.

Mary Dalrymple, John I amping, Pernando C. N. Pereiat and Vijay Sallswal. 1995. A deduetive accoum of quantilication in IJP(j. In Makolo Kantakawa. Christopher J. Pinón. and Hentene de Swart. editors. Quantifiers. Deduction. and Context. Center for the Study of Language and Information. Stantord. Callitomiat.

Mare Dymetman. 1992. Tramsformations de grammaires logiques et réversibilite en Traduction Automatique. These

is that it allows conlerring the "natural" lype e 110 an (in werseclive) adjective such as "hlack", or for a relative mod ther such as "hated hy peter", and also that there does not seen to exist any good reason why ype composition should be restricked of "functionally makching" types only, Semanlic type coercions abound in natural language as in the case of "glass etephant". "short win", etc., and these reyuire complex composition operations on the elements combined.

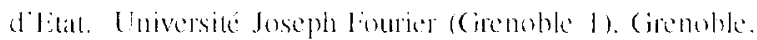
lrance

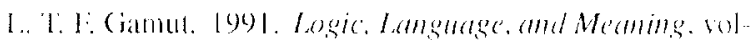

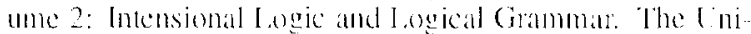
versily of Chicigo Press. (Micago).

Xiatorong Luang and Armin Fiedler. 1995, (ienerating mul. tilinguat proofs. In Richard Kitrodge adior. IICA/Workshoup on Multilingual Tet Genefation. palges 5.t 63. August.

Pierre Isabelle. Mare Dymetman. and lilliote Macklovitch. 1988. CIRTT"TLR: a transtation system for agricultural malket reports. In Proceedings of the 12 th herenational conference on Computational Lingustics. pages 261 266. Budapest. August.

Pience lsabelle. 1995, Un modele linguistique calculable pour la traduction automatigus bidirectionmelle. Ph. D. thesis. Université de Montréal.

Iohn I cevine. 1990. Pragma a llexible bielirectional dia logac systcm. In Proreedings. Lighl Nathonal conference on Artificial Intelligence. pages 964t (6).

Igor A. Mel'cuk. 1987. Dependency Sintar: Theory and Practice. State University of New York Press.

Igor Mel’üuk. 1981. Meaning-lext models. Joumal of Anthropology, 10:27 -62.

Cilyn V. Morrill. 1994. Type Logical Gimmar: Categorial logic of Signs. Kluwer Academic Publishers. Dordiecht. Ilollatukl.

Aarne Ranta. 1995. Type Theoretical Crammar. Oxford Universily Press. 